\title{
Cosmological perturbations from braneworld inflation with a Gauss-Bonnet term
}

\author{
Jean-Francois Dufaux ${ }^{1}$, James E. Lidsey ${ }^{2}$, Roy Maartens ${ }^{3}, \mathrm{M} \mathrm{Sami}^{4}$ \\ ${ }^{1}$ LPT, Université de Paris-Sud, 91405 Orsay, France \\ ${ }^{2}$ Astronomy Unit, School of Mathematical Sciences, \\ Queen Mary, University of London, London E1 4 NS, UK \\ ${ }^{3}$ Institute of Cosmology \& Gravitation, University of Portsmouth, Portsmouth PO1 2EG, UK \\ ${ }^{4}$ Inter-University Centre for Astronomy $\&$ Astrophysics, Pune, India
}

(Dated: October 29, 2018)

\begin{abstract}
Braneworld inflation is a phenomenology related to string theory that describes high-energy modifications to general relativistic inflation. The observable universe is a braneworld embedded in 5-dimensional anti de Sitter spacetime. When the 5-dimensional action is Einstein-Hilbert, we have a Randall-Sundrum type braneworld. The amplitude of tensor and scalar perturbations from inflation is strongly increased relative to the standard results, although the ratio of tensor to scalar amplitudes still obeys the standard consistency relation. If a Gauss-Bonnet term is included in the action, as a high-energy correction motivated by string theory, we show that there are important changes to the Randall-Sundrum case. We give an exact analysis of the tensor perturbations. They satisfy the same wave equation and have the same spectrum as in the Randall-Sundrum case, but the Gauss-Bonnet change to the junction conditions leads to a modified amplitude of gravitational waves. The amplitude is no longer monotonically increasing with energy scale, but decreases asymptotically after an initial rise above the standard level. Using an approximation that neglects bulk effects, we show that the amplitude of scalar perturbations has a qualitatively similar behaviour to the tensor amplitude. In addition, the tensor to scalar ratio breaks the standard consistency relation.
\end{abstract}

\section{INTRODUCTION}

In recent years, there has been considerable interest in the possibility that our observable four-dimensional (4D) universe may be viewed as a brane hypersurface embedded in a higher-dimensional bulk space. Of particular importance is the Randall-Sundrum (RS) model, where a single, positive-tension brane is embedded in a five-dimensional (5D) anti de Sitter $\left(\mathrm{AdS}_{5}\right)$ spacetime [1]. (For recent reviews, see Ref. [2].) Although the fifth dimension may be infinite in extent, the zero-mode of the $5 \mathrm{D}$ graviton, corresponding to $4 \mathrm{D}$ gravitational waves, is localized at low energies on the brane due to the warped geometry of the bulk. This property can also be understood within the context of the AdS/CFT correspondence [3], where the RS model is viewed as $4 \mathrm{D}$ gravity coupled to a conformal field theory (CFT) [4].

A natural extension of the RS model that is motivated by string/M theory considerations, is to include higherorder curvature invariants in the bulk action. Such terms arise in the AdS/CFT correspondence as next-to-leading order corrections to the CFT [5]. The Gauss-Bonnet (GB) combination of curvature invariants is of particular relevance in five dimensions, since it represents the unique combination that leads to second-order gravitational field equations in the bulk metric and since the field equations contain only linear second derivatives $[6]$. A GB term may also arise as the next-to-leading order correction in the heterotic string effective action, and it is ghost-free about flat spacetime [7]. Moreover, the graviton zero mode remains localized in the GB braneworld [8] and deviations from Newton's law at low energies are less pronounced than in the RS model [9].
From an observational perspective, there is now strong evidence that the very early universe underwent an epoch of accelerated (inflationary) expansion [10]. During inflation, light fields such as the graviton are quantummechanically excited and acquire nearly scale-invariant fluctuations 11]. The resulting spectrum of primordial gravitational waves could be detectable from its imprint on the polarization of the cosmic microwave background (CMB) 12. Such a detection would open a unique window into the physics of the very early universe. The evolution of gravitational waves during slow-roll inflation has been determined in the RS scenario 13. At high energies, the amplitude is enhanced relative to the standard result in $4 \mathrm{D}$ Einstein gravity. In view of the above developments, therefore, it is important to determine the properties of tensor perturbations generated during inflation in the GB braneworld. We show that significant changes to the RS case are introduced by the GB term, even when the GB corrections are very small relative to the Einstein-Hilbert terms.

\section{FIELD EQUATIONS}

For a 5D bulk with Einstein-Gauss-Bonnet gravity, containing a $4 \mathrm{D}$ brane, the gravitational action is

$$
\begin{aligned}
\mathcal{S}= & \frac{1}{2 \kappa_{5}^{2}} \int d^{5} x \sqrt{-{ }^{(5)} g}\left[-2 \Lambda_{5}+\mathcal{R}\right. \\
& \left.+\alpha\left(\mathcal{R}^{2}-4 \mathcal{R}_{a b} \mathcal{R}^{a b}+\mathcal{R}_{a b c d} \mathcal{R}^{a b c d}\right)\right] \\
& -\int_{\text {brane }} d^{4} x \sqrt{-g} \sigma
\end{aligned}
$$


where $x^{a}=\left(x^{\mu}, z\right), g_{a b}={ }^{(5)} g_{a b}-n_{a} n_{b}$ is the induced metric, with $n^{a}$ the unit normal to the brane, $\sigma(>0)$ is the brane tension, and $\Lambda_{5}(<0)$ is the bulk cosmological constant. The fundamental energy scale of gravity is the 5D scale $M_{5}$, where $\kappa_{5}^{2}=8 \pi / M_{5}^{3}$. The Planck scale $M_{4} \sim$ $10^{16} \mathrm{TeV}$ is an effective scale, describing gravity on the brane at low energies, and typically $M_{4} \gg M_{5}$.

The GB term may be thought of as the lowest-order stringy correction to the 5D Einstein-Hilbert action, with coupling constant $\alpha>0$. In this case, $\alpha\left|\mathcal{R}^{2}\right| \ll|\mathcal{R}|$, so that

$$
\alpha \ll \ell^{2}
$$

where $\ell$ is the bulk curvature scale, $|\mathcal{R}| \sim \ell^{-2}$. The RS type models are recovered for $\alpha=0$.

The 5D field equations following from the bulk action are

$$
\begin{aligned}
\mathcal{G}_{a b}= & -\Lambda_{5}{ }^{(5)} g_{a b}+\frac{\alpha}{2} \mathcal{H}_{a b} \\
\mathcal{H}_{a b}= & {\left[\mathcal{R}^{2}-4 \mathcal{R}_{c d} \mathcal{R}^{c d}+\mathcal{R}_{c d e f} \mathcal{R}^{c d e f}\right]{ }^{(5)} g_{a b} } \\
& -4\left[\mathcal{R} \mathcal{R}_{a b}-2 \mathcal{R}_{a c} \mathcal{R}^{c}{ }_{b}\right. \\
& \left.-2 \mathcal{R}_{a c b d} \mathcal{R}^{c d}+\mathcal{R}_{a c d e} \mathcal{R}_{b}{ }^{c d e}\right]
\end{aligned}
$$

The junction conditions at the brane, assuming mirror $\left(Z_{2}\right)$ symmetry, are [14]

$$
\begin{aligned}
K_{\mu \nu}-K g_{\mu \nu}= & -\frac{\kappa_{5}^{2}}{2}\left(T_{\mu \nu}-\sigma g_{\mu \nu}\right) \\
& -2 \alpha\left(Q_{\mu \nu}-\frac{1}{3} Q g_{\mu \nu}\right)
\end{aligned}
$$

where

$$
\begin{aligned}
Q_{\mu \nu}= & 2 K K_{\mu \alpha} K^{\alpha}{ }_{\nu}-2 K_{\mu \alpha} K^{\alpha \beta} K_{\beta \nu} \\
& +\left(K_{\alpha \beta} K^{\alpha \beta}-K^{2}\right) K_{\mu \nu}+2 K R_{\mu \nu} \\
& +R K_{\mu \nu}+2 K^{\alpha \beta} R_{\mu \alpha \beta \nu}-4 R_{\mu \alpha} K^{\alpha}{ }_{\nu}
\end{aligned}
$$

Here the curvature tenors are those of the 4D induced metric $g_{\mu \nu}, K_{\mu \nu}$ is the extrinsic curvature and $T_{\mu \nu}$ is the brane energy-momentum tensor. For a vacuum bulk, the conservation equations hold:

$$
\nabla^{\nu} T_{\mu \nu}=0
$$

An $\mathrm{AdS}_{5}$ bulk satisfies the 5D field equations, with

$$
\begin{aligned}
\overline{\mathcal{R}}_{a b c d} & =-\frac{1}{\ell^{2}}\left[{ }^{(5)} \bar{g}_{a c}{ }^{(5)} \bar{g}_{b d}-{ }^{(5)} \bar{g}_{a d}{ }^{(5)} \bar{g}_{b c}\right], \\
\overline{\mathcal{G}}_{a b} & =\frac{6}{\ell^{2}}{ }^{(5)} \bar{g}_{a b}=-\Lambda_{5}{ }^{(5)} \bar{g}_{a b}+\frac{\alpha}{2} \overline{\mathcal{H}}_{a b}, \\
\overline{\mathcal{H}}_{a b} & =\frac{24}{\ell^{4}}{ }^{(5)} \bar{g}_{a b} .
\end{aligned}
$$

It follows that

$$
\begin{aligned}
& \Lambda_{5}=-\frac{6}{\ell^{2}}+\frac{12 \alpha}{\ell^{4}} \\
& \frac{1}{\ell^{2}} \equiv \mu^{2}=\frac{1}{4 \alpha}\left[1-\sqrt{1+\frac{4}{3} \alpha \Lambda_{5}}\right],
\end{aligned}
$$

where we choose in Eq. (12) the branch with an RS limit, and $\mu$ is the energy scale associated with $\ell$. This reduces to the RS relation $1 / \ell^{2}=-\Lambda_{5} / 6$ when $\alpha=0$. Note that there is an upper limit to the GB coupling from Eq. (12):

$$
\alpha<\frac{\ell^{2}}{4}
$$

which in particular ensures that $\Lambda_{5}<0$.

A Friedman-Robertson-Walker (FRW) brane in an $\mathrm{AdS}_{5}$ bulk is a solution to the field and junction equations [15]. The modified Friedman equation on the (spatially flat) brane is [15, 16]

$$
\kappa_{5}^{2}(\rho+\sigma)=2 \sqrt{H^{2}+\mu^{2}}\left[3-4 \alpha \mu^{2}+8 \alpha H^{2}\right] .
$$

This may be rewritten in the useful form 17]

$$
\begin{aligned}
H^{2} & =\frac{1}{4 \alpha}\left[\left(1-4 \alpha \mu^{2}\right) \cosh \left(\frac{2 \chi}{3}\right)-1\right], \\
\kappa_{5}^{2}(\rho+\sigma) & =\left[\frac{2\left(1-4 \alpha \mu^{2}\right)^{3}}{\alpha}\right]^{1 / 2} \sinh \chi,
\end{aligned}
$$

where $\chi$ is a dimensionless measure of the energy density. Note that the limit in Eq. (13) is necessary for $H^{2}$ to be non-negative.

When $\rho=0=H$ in Eq. (14) we recover the expression for the critical brane tension which achieves zero cosmological constant on the brane,

$$
\kappa_{5}^{2} \sigma=2 \mu\left(3-4 \alpha \mu^{2}\right) .
$$

The effective 4D Newton constant is given by [8]

$$
\kappa_{4}^{2}=\frac{\mu}{\left(1+4 \alpha \mu^{2}\right)} \kappa_{5}^{2}
$$

When Eq. (2) holds, this implies $M_{5}^{3} \approx M_{4}^{2} / \ell$. Tabletop experiments to probe deviations from Newton's law currently imply $\ell \lesssim 0.1 \mathrm{~mm}$, so that $M_{5} \gtrsim 10^{5} \mathrm{TeV}$, and $\sigma \gtrsim(1 \mathrm{TeV})^{4}$, by Eqs. (2) and (17).

The modified Friedman equation (15), together with Eq. (16), shows that there is a characteristic GB energy scale,

$$
m_{\alpha}=\left[\frac{2\left(1-4 \alpha \mu^{2}\right)^{3}}{\alpha \kappa_{5}^{4}}\right]^{1 / 8}
$$

such that the GB high energy regime $(\chi \gg 1)$ is $\rho+$ $\sigma \gg m_{\alpha}^{4}$. If we consider the GB term in the action as a correction to RS gravity, then $m_{\alpha}$ is greater than the RS energy scale $m_{\sigma}=\sigma^{1 / 4}$, which marks the transition to RS high-energy corrections to $4 \mathrm{D}$ general relativity. By Eq. (17), this requires $3 \beta^{3}-12 \beta^{2}+15 \beta-2<0$ where $\beta \equiv 4 \alpha \mu^{2}$. Thus (to 2 significant figures),

$$
m_{\sigma}<m_{\alpha} \Rightarrow \alpha \mu^{2}<0.038
$$

which is consistent with Eq. (2). 
Expanding Eq. (15) in $\chi$, we find three regimes for the dynamical history of the brane universe:

the GB regime,

$$
\rho \gg m_{\alpha}^{4} \Rightarrow H^{2} \approx\left[\frac{\kappa_{5}^{2}}{16 \alpha} \rho\right]^{2 / 3}
$$

the RS regime,

$$
m_{\alpha}^{4} \gg \rho \gg \sigma \equiv m_{\sigma}^{4} \Rightarrow H^{2} \approx \frac{\kappa_{4}^{2}}{6 \sigma} \rho^{2},
$$

the $4 \mathrm{D}$ regime,

$$
\rho \ll \sigma \Rightarrow H^{2} \approx \frac{\kappa_{4}^{2}}{3} \rho .
$$

The GB regime, when the GB term dominates gravity at the highest energies, above the brane tension, can usefully be characterized as

$$
H^{2} \gg \alpha^{-1} \gg \mu^{2}, \quad H^{2} \propto \rho^{2 / 3} .
$$

The brane energy density should be limited by the quantum gravity limit, $\rho<M_{5}^{4}$, in the high-energy regime. By Eq. (21),

$$
\rho<M_{5}^{4} \Rightarrow H<\left(\frac{\pi M_{5}}{2 \alpha}\right)^{1 / 3} .
$$

In addition, since $\rho \gg m_{\alpha}^{4}$, we have [18]

$$
M_{5} \gg m_{\alpha} \Rightarrow \alpha \gg \frac{2}{\left(8 \pi M_{5}\right)^{2}} .
$$

Combining these two equations leads to

$$
m_{\alpha}^{4} \ll \rho<M_{5}^{4} \Rightarrow H \ll 4 \pi^{3 / 2} M_{5} .
$$

Comparing Eqs. (26) and (20), we also find that

$$
\ell \gg \frac{1}{8 \pi M_{5}}
$$

which is equivalent to $M_{4} \gg M_{5}$, since, by Eq. (18), we have $\ell \approx\left(M_{4} / M_{5}\right)^{2} M_{5}^{-1}$.

\section{BULK METRIC PERTURBATIONS}

A de Sitter brane in an $\mathrm{AdS}_{5}$ bulk is a solution to the GB field and junction equations. The brane has a constant Hubble rate $H$, and hence constant energy density $\rho>0$, which is added to the brane tension $\sigma$, thus effectively breaking the RS fine-tuning, as can be seen by comparing Eq. (14) with Eq. (17). Inflation in the extreme slow-roll regime may be modelled by this solution.

The bulk metric satisfies Eq. (9), and may be written in the form

$$
{ }^{(5)} d \bar{s}^{2}=A^{2}(z)\left[\gamma_{\mu \nu} d x^{\mu} d x^{\nu}+d z^{2}\right],
$$

where $\gamma_{\mu \nu}$ is the $4 \mathrm{D}$ de Sitter metric $\left(-d t^{2}+e^{2 H t} d \vec{x}^{2}\right)$, and the conformal warp factor is

$$
A(z)=\frac{H}{\mu \sinh H z},
$$

with $Z_{2}$ symmetry understood. The brane is at fixed position $z=z_{0}>0$, which we can choose so that $A\left(z_{0}\right)=$ 1 (i.e., $\sinh H z_{0}=H / \mu$ ). The horizon is at $z=\infty$.

Consider now the $5 \mathrm{D}$ spin-2 metric perturbations, ${ }^{(5)} \bar{g}_{a b} \rightarrow{ }^{(5)} \bar{g}_{a b}+\delta^{(5)} g_{a b}$, where $\delta^{(5)} g_{a b}$ is 5D transverse traceless. For these perturbations, Eq. (4) shows that $\delta \mathcal{H}^{a}{ }_{b}=0$, so that the wave equation for the perturbations is

$$
\delta \mathcal{R}^{a}{ }_{b}=0
$$

the same as in the RS case. This means that the bulk mode solutions for metric perturbations will be the same as in the RS case [13], but the GB junction conditions will introduce changes to the normalization and amplitudes of the modes, as discussed below.

In the gauge $\delta^{(5)} g_{a z}=0$, we can write the perturbed metric in the form

$$
{ }^{(5)} d s^{2}={ }^{(5)} d \bar{s}^{2}+A^{1 / 2} h_{\mu \nu} d x^{\mu} d x^{\nu},
$$

where the factor $A^{1 / 2}$ is introduced for later convenience. The perturbation may be decomposed into Kaluza-Klein (KK) modes,

$$
h_{\mu \nu}(x, z) \rightarrow h_{\mu \nu}^{(m)}(x) \phi_{m}(z),
$$

where integration (respectively sum) over the continuous (respectively discrete) modes is understood, and

$$
\gamma^{\mu \nu} h_{\mu \nu}^{(m)}=0=\nabla^{\mu} h_{\mu \nu}^{(m)}
$$

Here and below, $\nabla_{\mu}$ denotes the covariant derivative of the de Sitter metric $\gamma_{\mu \nu}$.

Because the brane is maximally symmetric, the wave equation separates in brane-based coordinates (as in the case $\alpha=013 \mid)$. The $4 \mathrm{D}$ part of the wave equation is (compare [19, 20])

$$
\square h_{\mu \nu}^{(m)}-2 H^{2} h_{\mu \nu}^{(m)}=m^{2} h_{\mu \nu}^{(m)},
$$

which describes the propagation of $4 \mathrm{D}$ massive modes on a de Sitter background. Here $\square=\nabla^{\mu} \nabla_{\mu}$. The spin- 2 quantity $h_{\mu \nu}^{(m)}$ encodes the 5 polarizations of the 5D graviton. This corresponds, from the viewpoint of a $4 \mathrm{D}$ observer, to 2 polarizations in a $4 \mathrm{D}$ tensor mode, 2 polarizations in a $4 \mathrm{D}$ vector mode (gravi-vector or gravi-photon), and 1 polarization in a 4D scalar mode (gravi-scalar). Each of these will have in general a zero-mode, i.e., a massless mode on the brane, and the massless modes satisfy the same junction condition as in the RS case (see below). However, for a single de Sitter brane, the zero mode perturbation $h_{\mu \nu}^{(0)}$ has only 2 independent degrees of freedom, corresponding to the usual $4 \mathrm{D}$ graviton. There 
are no massless modes for the gravi-vector and graviscalar 21, 22]; these degrees of freedom can be set to zero by the remaining gauge freedom on the brane [20].

The massive scalar and vector modes by contrast are not degenerate. They have the same behaviour in the bulk as the massive tensor modes. The massive modes of the $4 \mathrm{D}$ tensor perturbations satisfy the same bulk wave equation as in the RS case, but the junction condition at the brane is very different. The $4 \mathrm{D}$ tensor part of Eq. (5) gives

$$
\delta K^{\mu}{ }_{\nu} \propto \alpha \delta R^{\mu}{ }_{\nu}
$$

In the $\mathrm{RS}$ case $\alpha=0$, the right-hand side of this equation is zero, leading to the Neumann boundary condition, $\left(A^{-3 / 2} \phi_{m}\right)^{\prime}\left(z_{0}\right)=0$. On the brane, the perturbed Ricci tensor is given by 13 .

$$
2 \delta R_{\mu}{ }^{\nu}=\ddot{h}_{\mu}{ }^{\nu}+3 H \dot{h}_{\mu}{ }^{\nu}-e^{-2 H t} \partial_{i} \partial^{i} h_{\mu}{ }^{\nu} .
$$

Separating variables, it follows that $\delta R_{\mu}{ }^{\nu} \propto m^{2} h_{\mu}{ }^{\nu}$. Thus Eq. (35) shows that in the GB case, the boundary condition is of the form

$$
\left(A^{-3 / 2} \phi_{m}\right)^{\prime}\left(z_{0}\right) \propto \alpha m^{2} \phi_{m}\left(z_{0}\right) .
$$

The precise form of the boundary condition is given in Eq. (44) below.

\section{4D TENSOR PERTURBATIONS}

The wave equation for the massive tensor modes can be written in the form

$$
-\mathcal{D}_{+}\left[q(z) \mathcal{D}_{-} \phi_{m}(z)\right]=m^{2} w(z) \phi_{m}(z),
$$

where we define the operators

$$
\mathcal{D}_{ \pm}=\frac{d}{d z} \pm \frac{3}{2} \frac{A^{\prime}}{A}
$$

and the factors

$$
\begin{aligned}
q & =1-4 \alpha A^{-4}\left(A^{\prime 2}-A^{2} H^{2}\right), \\
w & =1-4 \alpha A^{-4}\left(A A^{\prime \prime}-A^{\prime 2}\right) .
\end{aligned}
$$

This form of the wave equation explicitly incorporates the GB junction condition. By Eq. (30),

$$
w=1-4 \alpha \mu^{2}-4 \alpha A^{-3}\left[A^{\prime}\right] \delta\left(z-z_{0}\right),
$$

where $\left[A^{\prime}\right]=2 A^{\prime}\left(z_{0}^{+}\right)=-2 \sqrt{\mu^{2}+H^{2}}$ is the jump in $A^{\prime}$ across the brane. Note from Eqs. (30), (40) and (42) that, for $z \neq z_{0}, q=w=1-4 \alpha \mu^{2}$. Thus for $z>z_{0}$, Eq. (38) reduces to the Schrödinger-type equation,

$$
\begin{aligned}
-\mathcal{D}_{+} \mathcal{D}_{-} \phi_{m} & =-\phi_{m}^{\prime \prime}+\left[\frac{15}{4} \frac{H^{2}}{\sinh ^{2} H z}+\frac{9}{4} H^{2}\right] \phi_{m} \\
& =m^{2} \phi_{m}
\end{aligned}
$$

exactly as in the RS case $\alpha=0[13,23]$.

The boundary condition for $\phi_{m}$ at $z=z_{0}$ is

$$
\mathcal{D}_{-} \phi_{m}\left(z_{0}^{+}\right)=-\alpha m^{2}\left[\frac{4 \sqrt{\mu^{2}+H^{2}}}{1-4 \alpha \mu^{2}}\right] \phi_{m}\left(z_{0}\right)
$$

and is of the form given in Eq. (37). This may be obtained by matching the distributional parts of Eq. (38). It is important to note that this boundary condition depends on the mass of the modes, $m^{2}$, due to the $\alpha$ corrections (the zero-mode, $m=0$, has the same boundary condition as in the RS case). As a result, the scalar product of the eigenmodes functional space has to include suitable boundary terms [24]. It may be checked that the eigenmodes resulting from Eqs. (43) and (44) are orthogonal with respect to the following scalar product:

$$
\begin{gathered}
\left(\phi_{m}, \phi_{n}\right)=2\left(1-4 \alpha \mu^{2}\right) \int_{z_{0}}^{\infty} d z \phi_{m}(z) \phi_{n}(z) \\
+8 \alpha \sqrt{\mu^{2}+H^{2}} \phi_{m}\left(z_{0}\right) \phi_{n}\left(z_{0}\right)=\delta(m, n) .
\end{gathered}
$$

The normalization in the last equality denotes a Kroneker symbol for the discrete modes and a Dirac distribution for the continuous ones. Note that Eq. (45) reduces formally to

$$
\left(\phi_{m}, \phi_{n}\right)=\int_{\mathrm{bulk}} d z w \phi_{m} \phi_{n}
$$

when $Z_{2}$-symmetry is imposed and the boundary term in Eq. (42) is taken into account. When $\alpha \geq 0$, the norm of the modes $\left\|\phi_{m}\right\|^{2}=\left(\phi_{m}, \phi_{m}\right)$ is always positive for the branch of solutions chosen in Eq. (12) and it reduces to the usual norm for $\alpha=0$.

With the orthonormal conditions in Eq. (45), the effective action for the metric perturbation, to second order, is:

$$
\begin{aligned}
S= & \frac{1}{2 \kappa_{5}^{2}}\left\{\frac { 1 } { 4 } \int d ^ { 4 } x \sqrt { - \gamma } \left[h^{(m) \mu \nu} \square h_{\mu \nu}^{(m)}\right.\right. \\
& \left.\left.-2 H^{2} h^{(m) \mu \nu} h_{\mu \nu}^{(m)}-m^{2} h^{(m) \mu \nu} h_{\mu \nu}^{(m)}\right]\right\},
\end{aligned}
$$

where the term in braces is the standard one for 4D (massive) gravitons on a de Sitter background.

We now consider the spectrum of modes resulting from Eqs. (43) and (44). There is a normalizable bound-state zero-mode, as in the RS case:

$$
\phi_{0}(z)=C A^{3 / 2}(z)
$$

where the real constant $C$ will be determined in the following. The asymptotic value of the Schrodinger potential in Eq. (43), i.e., $\frac{9}{4} H^{2}$, gives the threshold between the discrete and continuous spectra: $m^{2}>\frac{9}{4} H^{2}$, as in the RS case 13, 23]. For the massive modes in the continuous tower, the two linearly independent solutions of Eq. (43) oscillate with constant amplitude for $z \rightarrow \infty$. 
The boundary condition Eq. (44) gives $\phi_{m}(z)$ as a particular combination of these two solutions, for every $m$. These modes are normalizable as plane waves and form the continuous spectrum of Eqs. (43) and (44).

For $m^{2}<\frac{9}{4} H^{2}$ on the other hand, Eq. (43) admits only one independent normalizable solution for each $m$. The corresponding mode behaves as a decreasing exponential for $z \rightarrow \infty$. For $\alpha=0$, the only such mode which satisfies the junction condition is the massless mode, Eq. (47). In GB gravity however, this issue is more subtle because of the explicit dependence of the boundary condition Eq. (44) on $m^{2}$.

In order to see whether the junction conditions allow for discrete states other than the zero mode, it is convenient to introduce the new modes:

$$
\Phi_{m}(z)=\mathcal{D}_{-} \phi_{m}(z),
$$

which are the partners of the modes $\phi_{m}$ in supersymmetric quantum mechanics [25]. They have the same spectrum except for the zero-mode: $\Phi_{0}$ vanishes identically, by Eqs. (39) and (47). The wave equation for $\Phi_{m}$ is found by applying $\mathcal{D}_{-}$to Eq. (43):

$$
\begin{aligned}
-\mathcal{D}_{-} \mathcal{D}_{+} \Phi_{m} & =-\Phi_{m}^{\prime \prime}+\left[\frac{3}{4} \frac{H^{2}}{\sinh ^{2} H z}+\frac{9}{4} H^{2}\right] \Phi_{m} \\
& =m^{2} \Phi_{m} .
\end{aligned}
$$

The boundary condition follows from Eqs. (43) and (44),

$$
\Phi_{m}^{\prime}\left(z_{0}^{+}\right)=\left[\frac{1-4 \alpha \mu^{2}}{4 \alpha \sqrt{\mu^{2}+H^{2}}}+\frac{3}{2} \sqrt{\mu^{2}+H^{2}}\right] \Phi_{m}\left(z_{0}\right),
$$

for $\alpha \neq 0$, while $\Phi_{m}\left(z_{0}\right)=0$ for $\alpha=0$.

In particular, this boundary condition no longer involves the mass of the modes (and reduces to Dirichlettype for $\alpha=0$ ). This is the essential property we need. Multiplying Eq. (49) by $\Phi_{m}$ and integrating by parts, we find that

$$
\begin{gathered}
\left(m^{2}-\frac{9}{4} H^{2}\right) \int_{z_{0}}^{\infty} d z \Phi_{m}^{2}=\frac{3}{4} H^{2} \int_{z_{0}}^{\infty} d z \frac{\Phi_{m}^{2}}{\sinh ^{2} H z} \\
+\int_{z_{0}}^{\infty} d z \Phi_{m}^{\prime 2}+\left[-\Phi_{m} \Phi_{m}^{\prime}\right]_{z_{0}}^{\infty}
\end{gathered}
$$

Consider now a would-be normalizable mode $\phi_{m}$ with $m<\frac{3}{2} H$. Its partner $\Phi_{m}$ must decrease exponentially when $z \rightarrow \infty$, as does $\phi_{m}$. The corresponding upper boundary term at infinity in Eq. (51) therefore vanishes. The lower boundary term on the brane, by Eq. (50), is positive for the minus branch of solutions, defined in Eq. (12), and for $\alpha \geq 0$. It vanishes for $\alpha=0$. Thus in this case, the right-hand side of Eq. (51) is positive, while the left-hand side is negative. This can be satisfied only for $\Phi_{m}=0$, i.e., for $m=0$. We therefore conclude that the spectrum of KK modes consists only of

- the massless bound-state zero-mode,
- a continuum of states with $m>\frac{3}{2} H$,

as in the RS case $\alpha=0$ [13, 23.

This feature is crucial for discussing stability issues as well as the gravitational waves produced along the brane. In particular, the spectrum rules out the existence of $4 \mathrm{D}$ massive gravitons with $m^{2}<2 H^{2}$ in Eq. (34), which would have signalled a classical instability of the model [19] (see also Ref. 26] for a recent discussion in the braneworld context). It has been shown that a mass gap for de Sitter branes is quite generic in Einstein gravity 20]. In particular it still holds if a second $Z_{2}$-symmetric brane is introduced in the background, Eq. (29), say at $z=z_{2}>z_{0}$. We just note here however that we can not reach the same conclusion in Gauss-Bonnet gravity. In particular, the argument above would fail in this case, because the new boundary term at $z=z_{2}<\infty$ in Eq. (51) would then be negative (while it still vanishes for $\alpha=0$ ). In fact, if we solve Eq. (43) explicitly and impose Eq. (44), we can show that tachyonic modes with $m^{2}<0\left(<2 H^{2}\right)$ may exist for the 2-brane system with $\alpha>0$ (as well as for the 1-brane case with $\alpha<0$ ). This system may therefore suffer from the same spin-2 tachyonic instability present for Minkowski branes 27]. (Note that the tachyonic instability in the case of two de Sitter branes with Einstein gravity [22, 28] is a spin- 0 radion mode.)

\section{AMPLITUDE OF THE ZERO-MODE}

We can now estimate the spectrum of graviton fluctuations generated in de Sitter inflation on the brane, by treating each mode as a quantum field in four dimensions, as in the RS case [13, 20] (see Refs. 29, 30] for a five-dimensional approach).

For $m^{2}>\frac{9}{4} H^{2}$, the massive modes are strongly suppressed on large scales and remain in their vacuum state [13, 20]. These modes can therefore be neglected in the following. However, the zero-mode is over-damped and acquires a spectrum of classical perturbations on super-horizon scales. For $m^{2}=0$, the effective action Eq. (46) has the standard form of $4 \mathrm{D}$ general relativity, except for the overall factor $\kappa_{5}^{2}$ instead of $\kappa_{4}^{2}$, which rescales the amplitude of quantum fluctuations in $h_{\mu \nu}^{(0)}$ accordingly [13]. Thus the amplitude of gravitational waves produced on super-horizon scales on the brane is given by

$$
A_{T}^{2}=\kappa_{5}^{2} \phi_{0}^{2}\left(z_{0}\right)\left(\frac{H}{2 \pi}\right)^{2}
$$

The normalization of the discrete zero-mode, $\phi_{0}\left(z_{0}\right)=C$, introduces further rescaling relative to the $4 \mathrm{D}$ result. By Eqs. (45) and (47), the condition $\left(\phi_{0}, \phi_{0}\right)=1$ gives:

$$
C^{-2}=\frac{\left(1+4 \alpha \mu^{2}\right)}{\mu} F_{\alpha}^{-2}(H / \mu),
$$


where we used Eq. (18), and where

$$
F_{\alpha}^{-2}(x)=\sqrt{1+x^{2}}-\left(\frac{1-4 \alpha \mu^{2}}{1+4 \alpha \mu^{2}}\right) x^{2} \sinh ^{-1} \frac{1}{x} .
$$

This generalizes the function $F_{0}(x)$ found for the RS case [13]. When $x \equiv H / \mu \rightarrow 0$, we have $F_{\alpha} \rightarrow 1$; the amplitude of the normalized zero-mode on a Minkowski brane measures the ratio between the effective $4 \mathrm{D}$ Newton constant $\kappa_{4}^{2}$, and the $5 \mathrm{D}$ constant $\kappa_{5}^{2}$.

The modified tensor amplitude is therefore

$$
A_{T}^{2}=\kappa_{4}^{2}\left(\frac{H}{2 \pi}\right)^{2} F_{\alpha}^{2}(H / \mu)
$$

and the correction to standard 4D general relativity lies in the last factor:

$$
F_{\alpha}^{2}=\frac{A_{T}^{2}}{\left[A_{T}^{2}\right]_{4 \mathrm{D}}} .
$$

This correction depends on the GB coupling $\alpha$ and on the energy scale at which inflation occurs, relative to the $5 \mathrm{D}$ curvature scale $\mu$, and it reduces to the result of Ref. 13. for the RS case $\alpha=0$. (The correction to the $4 \mathrm{D}$ result may also be expressed via an effective Planck mass during inflation, following Ref. [20].)

The GB term introduces significant corrections to the RS case. In the GB regime, as characterized by Eq. (24), we have

$$
F_{\alpha}^{2}(H / \mu) \approx \frac{\left(1+4 \alpha \mu^{2}\right)}{8 \alpha \mu^{2}}\left(\frac{H}{\mu}\right)^{-1}
$$

while the RS case $\alpha=0$ yields

$$
F_{\alpha=0}^{2}(H / \mu) \approx \frac{3}{2} \frac{H}{\mu} .
$$

Thus the GB term suppresses tensor perturbations relative to the $4 \mathrm{D}$ result, at high energies, unlike the RS case where the tensor amplitude is strongly enhanced. If we consider the GB term as a perturbative correction to the Einstein-Hilbert 5D action, then $\beta \equiv 4 \alpha \mu^{2} \ll 1$, and there is an RS regime as the energy drops (but remains above the brane tension). Thus we expect that the tensor amplitude is enhanced at lower energies (RS regime) and suppressed at higher energies (GB regime), so that there is a maximum at intermediate energies. This qualitative behaviour is confirmed in Figs. 1 and 2 .

The quantum gravity limit $\rho<M_{5}^{4}$ imposes an upper limit on the energy scale $x$; by Eqs. (18) and (27),

$$
x \equiv \frac{H}{\mu} \ll\left(\frac{M_{4}}{M_{5}}\right)^{2} .
$$

There is a range of energies, $0<H<H_{0}$, where $H_{0}$ is the solution to $F_{\alpha}^{2}\left(H_{0}\right)=1$, such that $F_{\alpha}^{2}(H)>1$, i.e., such that the amplitude of gravitational waves from GB

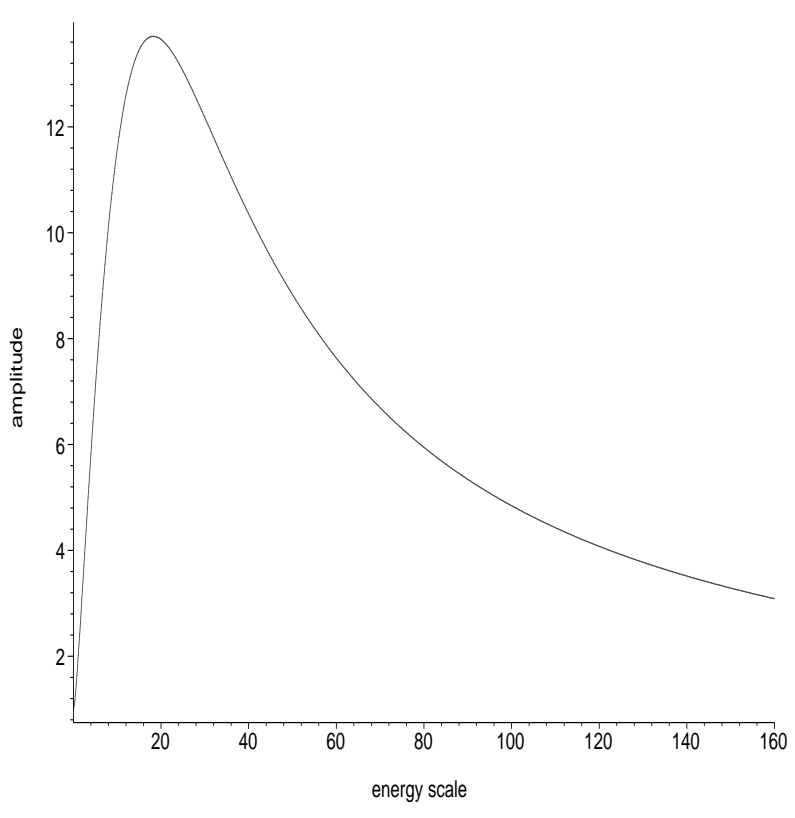

FIG. 1: The dimensionless amplitude $F_{\alpha}^{2}$ of the tensor zeromode relative to the $4 \mathrm{D}$ general relativity result, plotted against the dimensionless energy scale of inflation, $H / \mu$. (The Gauss-Bonnet coupling is given by $\beta \equiv 4 \alpha \mu^{2}=10^{-3}$.)

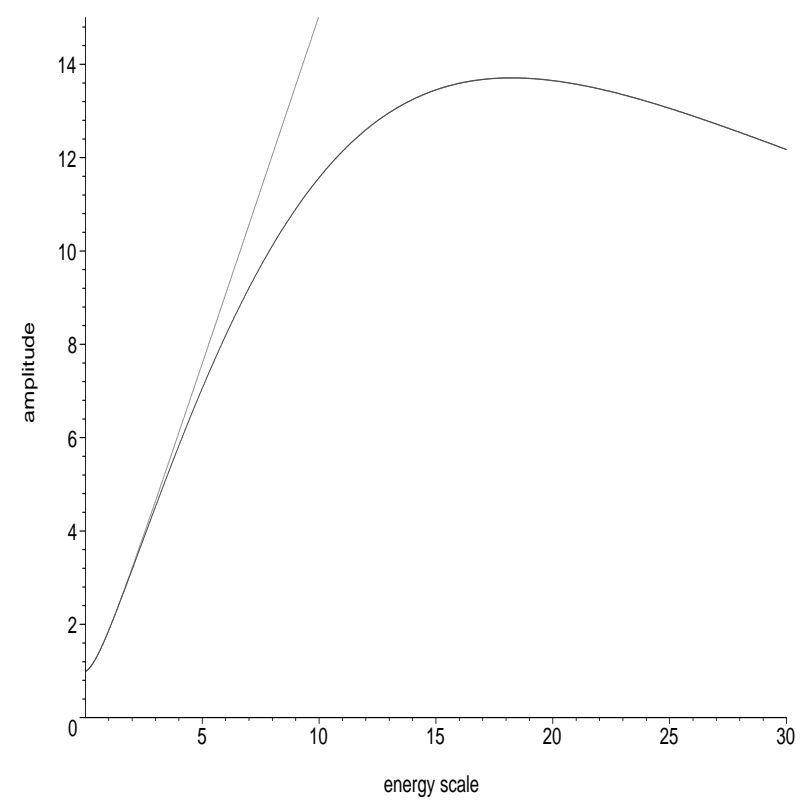

FIG. 2: As in Fig. 1, but with the upper curve showing the Randall-Sundrum case $\alpha=0$. 
inflation is greater than the standard 4D amplitude. By Eqs. (2) and (57),

$$
0<H<H_{0} \approx \frac{1}{8 \alpha \mu} \Rightarrow F_{\alpha}^{2}>1 \text {. }
$$

Changing the value of $\alpha$ changes the location and height of the maximum, and the value of $H_{0}$, but the maximum always has $F_{\alpha}^{2}>1$. In all cases, $F_{\alpha}(0)=1$, and the asymptotic behaviour as $x \rightarrow \infty$ is $F_{\alpha}^{2} \sim x^{-1}$, as given by Eq. (57). The maximum of $F_{\alpha}^{2}$ increases as $\alpha$ decreases, and so does the range $H_{0}$ of energies for which the amplitude exceeds the $4 \mathrm{D}$ result. In the RS limit $\alpha \rightarrow 0$, there is no maximum: $F_{0}^{2}$ is monotonically increasing without bound for $x \rightarrow \infty$, as illustrated in Fig. 2 .

The maximum gravitational-wave amplitude relative to the standard $4 \mathrm{D}$ result for $\alpha>0$ is given by

$$
\left(F_{\alpha}^{2}\right)_{\max }=\frac{A_{T, \max }^{2}}{\left[A_{T}^{2}\right]_{4 \mathrm{D}}}=\frac{\left(1+4 \alpha \mu^{2}\right) \sqrt{1+H_{\mathrm{m}}^{2} / \mu^{2}}}{1+4 \alpha \mu^{2}+4 \alpha H_{\mathrm{m}}^{2}},
$$

where the critical inflation energy scale $H_{\mathrm{m}}$ is determined by the root of the equation

$$
\sqrt{1+x_{\mathrm{m}}^{2}} \sinh ^{-1} \frac{1}{x_{\mathrm{m}}}=\frac{1}{1-4 \alpha \mu^{2}} .
$$

\section{THE TENSOR TO SCALAR RATIO}

It is well known that in standard, slow-roll inflation driven by a single inflaton field, the scalar and tensor perturbations are not independent, but are instead related by a consistency relation. (For a review, see, e.g., Ref. 31$]$ ). To lowest order in the slow-roll approximation, the ratio of the tensor to scalar perturbations is given by

$$
\frac{A_{T}^{2}}{A_{S}^{2}}=-\frac{1}{2} n_{T}
$$

where $n_{T} \equiv d \ln A_{T}^{2} / d \ln k$ represents the tilt of the tensorial spectrum and $k$ is comoving wavenumber. An identical relation holds in 4D scalar-tensor and other generalized Einstein theories [32], and also in the RS scenario [33, 34], and in a 5D braneworld model where the radion field is stabilized 35]. Formally, the degeneracy in the braneworld models arises because the function that parametrizes the corrections to the gravitational wave amplitude satisfies a particular first-order differential equation [33, 36].

Given the potential importance of the consistency relation as a way of reducing the number of independent inflationary parameters, and of testing the inflationary scenario, it is important to investigate whether the above degeneracy is lifted when GB effects are included in the bulk action as a correction to the RS model. Furthermore, the relative contribution of tensor perturbations to CMB anisotropies is also an important quantity for constraining inflationary models [37], and we will consider how the GB term affects this. In the RS case, although both tensor and scalar perturbations are enhanced, the tensors are enhanced less and thus the relative tensor contribution is suppressed in comparison with the standard case. First we need to compute the scalar perturbation amplitude $A_{S}$.

\section{A. Scalar perturbations from GB brane inflation}

We assume that there is no scalar zero-mode contribution from bulk metric perturbations (5D gravitons) during inflation, and that the massive scalar KK modes may be neglected in inflation. The latter is true in the exact de Sitter inflation case, as discussed above. The scalar massive modes may be ignored, since they are heavy $\left(m>\frac{3}{2} H\right)$ and stay in their vacuum state during inflation, both for the RS case and the GB generalization. For more general inflationary expansion, it may not be realistic to ignore the massive modes, but in the extreme slow-roll limit, it may be a reasonable approximation to neglect the bulk metric perturbations. In this approximation we can take over the standard $4 \mathrm{D}$ results that do not depend on the standard Friedman equation, as in the RS case [38].

Conservation of energy-momentum on the brane, Eq. (7), implies that the adiabatic matter curvature perturbation $\zeta$ on a uniform density hypersurface is conserved on large scales, independently of the gravitational physics [39]. Consequently, the amplitude of a given mode that re-enters the Hubble radius after inflation is given by $A_{S}^{2}=H^{4} /\left(2 \pi^{2} \dot{\varphi}^{2}\right)$. Here and in similar expressions in this Section, equality is to be understood as equality at the lowest order in the slow-roll approximation. (The normalization is chosen such that $\left.A_{S}^{2}=2\left\langle\zeta^{2}\right\rangle.\right)$ In this limit, the scalar field equation, $\dot{\varphi}=-V^{\prime}(\varphi) / 3 H$, implies that the amplitude of scalar (density) perturbations is given by

$$
A_{S}^{2}=\frac{9}{2 \pi^{2}} \frac{H^{6}}{V^{\prime 2}}
$$

Using Eqs. (15), (16), (17) and (18), we can write this in terms of the standard result, $\left[A_{S}^{2}\right]_{4 \mathrm{D}}=\kappa_{4}^{6} V^{3} / 6 \pi^{2} V^{\prime 2}$, as follows:

$$
A_{S}^{2}=\left[A_{S}^{2}\right]_{4 \mathrm{D}} G_{\alpha}^{2}(H / \mu)
$$

where

$$
G_{\alpha}^{2}(x)=\left[\frac{3(1+\beta) x^{2}}{2 \sqrt{1+x^{2}}\left(3-\beta+2 \beta x^{2}\right)+2(\beta-3)}\right]^{3},
$$

with $\beta \equiv 4 \alpha \mu^{2}$.

The scalar spectral index, $n_{S}-1 \equiv d \ln A_{S}^{2} /\left.d \ln k\right|_{k=a H}$ can be expressed in terms of the slow-roll parameters, $\epsilon \equiv-\dot{H} / H^{2}$ and $\eta \equiv V^{\prime \prime} / 3 H^{2}$, such that [40]

$$
n_{S}-1=-6 \epsilon+2 \eta
$$




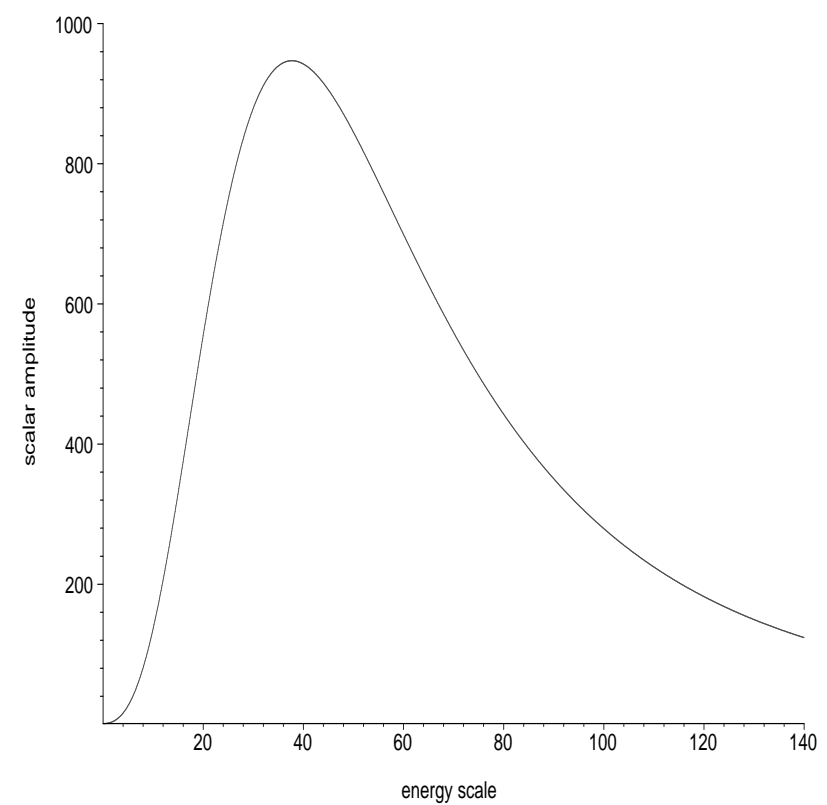

FIG. 3: The dimensionless amplitude $G_{\alpha}^{2}$ of density perturbations relative to the $4 \mathrm{D}$ general relativity result, plotted against the dimensionless energy scale of inflation, $H / \mu$. (The Gauss-Bonnet coupling is given by $\beta \equiv 4 \alpha \mu^{2}=10^{-3}$.)

where

$$
\begin{aligned}
\frac{\epsilon}{\epsilon_{\mathrm{RS}}} & =\frac{2(1-\beta)^{4} \sinh \frac{2}{3} \chi \tanh \chi \sinh ^{2} \chi}{9(1+\beta)(3-\beta)\left[(1-\beta) \cosh \frac{2}{3} \chi-1\right]^{2}}, \\
\frac{\eta}{\eta_{\mathrm{RS}}} & =\frac{2(1-\beta)^{3} \sinh ^{2} \chi}{3(1+\beta)(3-\beta)\left[(1-\beta) \cosh \frac{2}{3} \chi-1\right]},
\end{aligned}
$$

and $\epsilon_{\mathrm{RS}} \equiv 2 \sigma V^{\prime 2} /\left(\kappa_{4}^{2} V^{3}\right)$ and $\eta_{\mathrm{RS}} \equiv 2 \sigma V^{\prime \prime} /\left(\kappa_{4}^{2} V^{2}\right)$ are the corresponding RS slow-roll parameters [38].

As in the case of tensor perturbations, the scalar perturbations with GB corrections behave very differently compared to the RS case. At high energies, the GB term again leads to a suppression of scalar perturbations relative to the standard result. In the GB regime, as characterized by Eq. (24), we have

$$
G_{\alpha}^{2} \approx \frac{27}{64}\left(\frac{1+\beta}{\beta}\right)^{3} \frac{1}{x^{3}} .
$$

By contrast, in the RS case, scalar perturbations are strongly enhanced at high energies. Thus we have a similar qualitative behaviour to the tensor case: there is an $\mathrm{RS}$ regime of amplification at lower energies, and a GB regime of suppression, with a maximum at intermediate energies. The qualitative behaviour of the dimensionless amplitude of scalar perturbations is shown in Fig. 3.

Furthermore, the different scaling of the scalar and tensor amplitudes at high energies leads to another intriguing difference from the RS case. In the RS case,

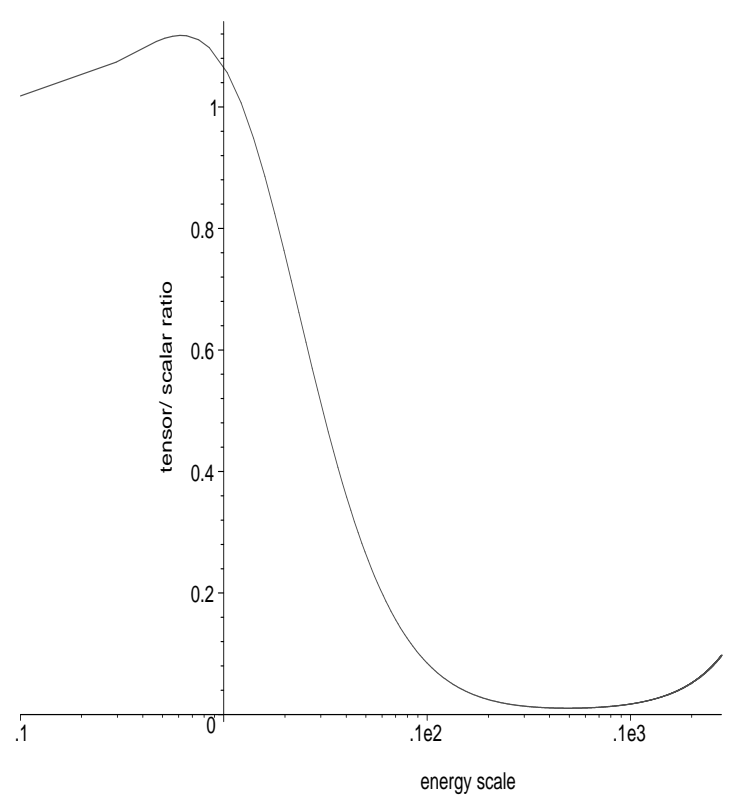

FIG. 4: The ratio of tensor to scalar perturbations, $R=$ $A_{T}^{2} / A_{S}^{2}$, relative to the $4 \mathrm{D}$ general relativity ratio, plotted against the dimensionless energy scale of inflation, $\log (H / \mu)$. (The Gauss-Bonnet coupling is given by $\beta \equiv 4 \alpha \mu^{2}=10^{-3}$.)

tensors are enhanced less strongly than scalars, so that the tensor/ scalar ratio $R=A_{T}^{2} / A_{S}^{2}$ is suppressed in comparison with the standard case. When there is a GB term, Eqs. (57) and (70) show that the scalars are more strongly suppressed at high energies than the tensors, so that the tensor/scalar ratio is enhanced at high energies. At lower and intermediate energies the ratio is a complicated function of $x$, given the interplay between RS and GB effects. It follows from Eqs. (54) and (66) that

$$
\begin{aligned}
& \frac{R}{R_{4 \mathrm{D}}}= \\
& \frac{\left[2 \sqrt{1+x^{2}}\left(3-\beta+2 \beta x^{2}\right)+2(\beta-3)\right]^{3}}{27(1+\beta)^{2} x^{6}\left[(1+\beta) \sqrt{1+x^{2}}-(1-\beta) x^{2} \sinh ^{-1} x^{-1}\right]}
\end{aligned}
$$

where $R_{4 \mathrm{D}}=\left[A_{T}^{2} / A_{S}^{2}\right]_{4 \mathrm{D}}$. The ratio of tensor to scalar amplitudes, relative to the standard ratio, has a maximum at low energies, a minimum at high energies, and grows like $x^{2}$ at very high energies. This is illustrated in Fig. 4.

\section{B. Consistency relation}

The consistency relation for the GB braneworld is derived by differentiating the gravitational wave amplitude, Eq. (55), with respect to comoving wavenumber $k(\varphi)=a(\varphi) H(\varphi)$. In the extreme slow-roll limit, variations in the Hubble parameter are negligible relative to 
changes in the scale factor. This implies that the tensor spectral index can be expressed as

$$
n_{T}=-\frac{d \ln \left(x F_{\alpha}\right)^{-2}}{d \ln x} \frac{a}{H} \frac{d H}{d a} .
$$

The GB braneworld correction to the gravitational amplitude, Eq. (54), satisfies an important first-order differential equation:

$$
\frac{d}{d \ln x}\left[\ln \left(x F_{\alpha}\right)^{-2}\right]=-\frac{2 F_{\alpha}^{2}\left[1+\beta\left(1+x^{2}\right)\right]}{(1+\beta) \sqrt{1+x^{2}}} .
$$

Furthermore, the scalar field equation can be expressed in the form

$$
a \frac{d H}{d a}=-\frac{d H}{d V} \frac{V^{\prime 2}}{3 H^{2}} .
$$

Hence, substituting Eqs. (55), (64), (73) and (774) into Eq. (72) implies that the tensor to scalar ratio is given by

$$
\frac{A_{T}^{2}}{A_{S}^{2}}=-\frac{Q}{2} n_{T}
$$

where

$$
Q^{-1}=\frac{6}{\kappa_{4}^{2}} \frac{\left[1+\beta\left(1+x^{2}\right)\right]}{(1+\beta) \sqrt{1+x^{2}}} H \frac{d H}{d V} .
$$

The function $Q(H)$ determines to what extent the degeneracy of the consistency equation is lifted in GB braneworld inflation and we therefore refer to it as the "degeneracy factor". For our normalization conventions, it takes the value $Q=1$ in the standard and also the RS inflationary scenarios.

Differentiating Eqs. (15) and (16) with respect to $\chi$, Eq. (176) then implies that

$$
Q=\frac{(1-\beta) \cosh \chi}{\left[1+2(1-\beta) \sinh ^{2}(\chi / 3)\right] \cosh (\chi / 3)},
$$

where the result has been simplified by employing Eq. (18). Using the identity $\cosh \chi+\cosh (\chi / 3)=$ $2 \cosh (2 \chi / 3) \cosh (\chi / 3)$, we find that the degeneracy factor takes the simple form:

$$
Q=\frac{1+\beta+2 \beta x^{2}}{1+\beta+\beta x^{2}} .
$$

We may conclude immediately from Eq. (78) that GB effects lift the degeneracy of the consistency equation, since the factor $Q$ depends directly on the energy scale, $x$, corresponding to the time when the observable modes went beyond the Hubble radius during inflation. The standard form of the consistency equation $(Q=1)$ is recovered for $\alpha=0$, and also in the limit $\beta x^{2} \equiv 4 \alpha \mu^{2} x^{2} \ll 1$, corresponding to the regimes of Eqs. (22) and (23) in the history of the GB braneworld. However, in the GB regime of Eq. (24), the asymptotic

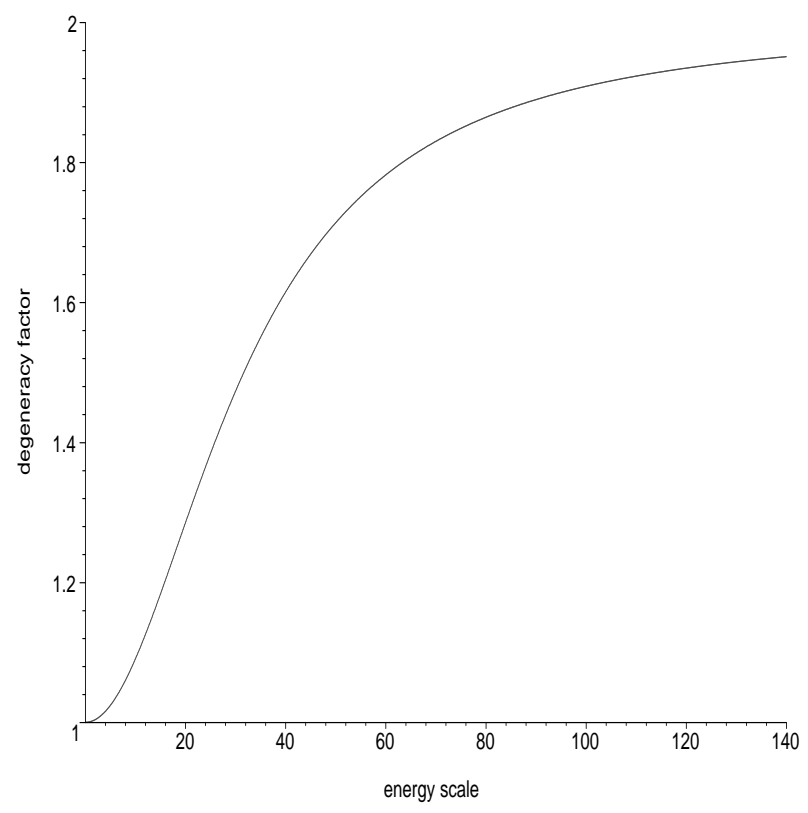

FIG. 5: The tensor/ scalar degeneracy factor $Q$, plotted against the dimensionless energy scale of inflation, $H / \mu$. (The Gauss-Bonnet coupling is given by $\beta \equiv 4 \alpha \mu^{2}=10^{-3}$.)

form of the degeneracy factor is $Q \rightarrow 2$. In this limit the consistency equation is given by

$$
\frac{A_{T}^{2}}{A_{S}^{2}}=-n_{T}
$$

The behaviour of $Q$ as a function of energy scale is shown in Fig. 5.

It is interesting that Eq. (79) is independent not only of the specific form of the inflaton potential, but also of the parameters of the model, specifically the brane tension, $\sigma$, the bulk cosmological constant, $\Lambda_{5}$, and the GB parameter, $\alpha$. As in the standard $4 \mathrm{D}$ and the $5 \mathrm{D}$ RS scenarios, the consistency relation can be expressed entirely in terms of observable parameters and, in this sense, Eq. (79) may be viewed as a model-independent observable signature of GB braneworld inflation in the high energy limit, Eq. (21).

\section{CONCLUSIONS}

Brane inflation offers a phenomenology that allows us to explore some of the cosmological implications of ideas arising from string and $\mathrm{M}$ theory. The effects on inflationary perturbations from the extra dimensional nature of gravity introduce new features that need to be computed and then subjected to the constraints from high-precision cosmological data. Here we have concentrated on computing the corrections to the standard results for tensor 
and scalar perturbations that are generated during slowroll inflation at energies where brane effects become dominant. This has previously been done for the RandallSundrum braneworld, based on 5-dimensional Einstein gravity. We have introduced a Gauss-Bonnet term, since string theory arguments indicate that this term is a high-energy perturbative correction to the gravitational action. This correction leads to significant qualitative changes, even in the perturbative regime $\beta \equiv 4 \alpha \mu^{2} \ll 1$.

For the tensor perturbations, we have given an exact analysis, including the $5 \mathrm{D}$ modes. The wave equation and its fundamental solutions are not changed by the GB term. The spectrum contains a normalizable zeromode and a continuous tower of massive modes after a mass gap, $m>\frac{3}{2} H$, as in the RS case. The massive modes are not excited during inflation, as in the $\mathrm{RS}$ case. However, the GB term changes the boundary conditions at the brane, and therefore changes the normalization of the zero-mode, as shown by Eq. (54):

$$
\frac{A_{T}^{2}}{\left[A_{T}^{2}\right]_{4 \mathrm{D}}}=\left[\sqrt{1+x^{2}}-\left(\frac{1-\beta}{1+\beta}\right) x^{2} \sinh ^{-1} \frac{1}{x}\right]^{-2} .
$$

This leads in the GB regime to a suppression of tensor perturbations relative to the standard result, unlike the enhancement that arises in the RS case $\beta=0$.

For the scalar perturbations, we used an approximation where bulk perturbations decouple from the density perturbations. We showed that the GB modifications to the Friedman equation lead to a significant change from the RS case, as given by Eq. (66):

$$
\frac{A_{S}^{2}}{\left[A_{S}^{2}\right]_{4 \mathrm{D}}}=\left[\frac{3(1+\beta) x^{2}}{2 \sqrt{1+x^{2}}\left(3-\beta+2 \beta x^{2}\right)+2(\beta-3)}\right]^{3} \text {. }
$$

These perturbations are again suppressed in the GB regime relative to the standard result, unlike the $\mathrm{RS}$ enhancement.
Because the scalar suppression is stronger than the tensor suppression in the GB regime, the relative tensor contribution, as a fraction of the scalar amplitude, $R=A_{T}^{2} / A_{S}^{2}$, is enhanced in the GB regime, in comparison with the standard result. This is shown by Eq. (71):

$$
\begin{aligned}
& \frac{R}{R_{4 \mathrm{D}}}= \\
& \frac{\left[2 \sqrt{1+x^{2}}\left(3-\beta+2 \beta x^{2}\right)+2(\beta-3)\right]^{3}}{27(1+\beta)^{2} x^{6}\left[(1+\beta) \sqrt{1+x^{2}}-(1-\beta) x^{2} \sinh ^{-1} x^{-1}\right]} .
\end{aligned}
$$

By contrast, in the RS case the relative tensor contribution is suppressed.

Furthermore, the consistency relation between the tensor/ scalar ratio and the tensor spectral tilt is different in the GB case, i.e.,

$$
R=-\left(\frac{1+\beta+2 \beta x^{2}}{1+\beta+\beta x^{2}}\right) \frac{n_{T}}{2}
$$

by Eq. (78). The RS model by contrast has the same consistency relation as the standard case, $R=-\frac{1}{2} n_{T}$.

Our results provide a basis on which to confront the GB braneworld with observational constraints, and this is under investigation.

\section{Acknowledgements:}

We thank Pierre Binetruy, Christos Charmousis, Naresh Dadhich, Stephen Davis, Lev Kofman, David Langlois, Jihad Mourad, Sergei Odintsov, Renaud Parentani, Danielle Steer and David Wands for helpful discussions. The work of RM is supported by PPARC.
[1] L. Randall and R. Sundrum, Phys. Rev. Lett. 83, 4690 (1999) hep-th/9906064.

[2] P. Brax and C. van de Bruck, Class. Quantum Grav. 20, R201 (2003) hep-th/0303095; R. Maartens, gr-qc/0312059 P. Brax, C. van de Bruck, and A-C. Davis, hep-th/0404011

[3] J. M. Maldacena, Adv. Theor. Math. Phys. 2, 231 (1998) hep-th/9711200; E. Witten, Adv. Theor. Math. Phys. 2, 505 (1998) hep-th/9803131; S. Gubser, I. Klebanov, and A. Polyakov, Phys. Lett. B428, 105 (1998) hep-th/9802109; O. Aharony, S. Gubser, J. Maldacena, H. Ooguri, and Y. Oz, Phys. Rep. 323, 183 (2000) hep-th/9905111.

[4] S. W. Hawking, T. Hertog, and H. Reall, Phys. Rev. D62 043501 (2000) hep-th/0003052; M. J. Duff and J. T. Liu, Phys. Rev. Lett. 85, 2052 (2000) hep-th/0003237; S. Nojiri, S. D. Odintsov, and S. Zerbini, Phys. Rev. D62, 064006 (2000) hep-th/0001192; S. No- jiri and S. Odintsov, Phys. Lett. B484, 119 (2000) hep-th/0004097; S. Nojiri and S. D. Odintsov, J. High Energy Phys. 07, 049 (2000) hep-th/0006232; L. Anchordoqui, C. Nunez, and K. Olsen, J. High Energy Phys. 10, 050 (2000) hep-th/0007064; S. Nojiri and S. Odintsov, Phys. Lett. B494, 135 (2000) hep-th/0008160; S. Gubser, Phys. Rev. D63, 084017 (2001) hep-th/9912001; T. Shiromizu and D. Ida, Phys. Rev. D64, 044015 (2001) hep-th/0102035; M. Cvetic, S. Nojiri and S. D. Odintsov, Nucl. Phys. B628, 295 (2002) hep-th/0112045; J. E. Lidsey, S. Nojiri and S. D. Odintsov, J. High Energy Phys. 06, 026 (2002) hep-th/0202198.

[5] A. Fayyazuddin and M. Spalinski, Nucl. Phys. B535, 219 (1998) hep-th/9805096; O. Aharony, A. Fayyazuddin, and J. Maldacena, J. High Energy Phys. 07, 013 (1998) hep-th/9806159.

[6] D. Lovelock, J. Math. Phys. 12, 498 (1971); N. Deru- 
elle and J. Madore, Mod. Phys. Lett. A1, 237 (1986); N. Deruelle and L. Farina-Busto, Phys. Rev. D41, 3696 (1990).

[7] D. G. Boulware and S. Deser, Phys. Rev. Lett. 55, 2656 (1985); B. Zwiebach, Phys. Lett. B156, 315 (1985); A. Sen, Phys. Rev. Lett. 55, 1846 (1985); R. R. Metsaev and A. A. Tseytlin, Nucl. Phys. B293, 385 (1987).

[8] N. E. Mavromatos and J. Rizos, Phys. Rev. D62, 124004 (2000) hep-th/0008074; I. P. Neupane, J. High Energy Phys. 09, 040 (2000) hep-th/0008190; I. P. Neupane, Phys. Lett. B512, 137 (2001) hep-th/0104226; K. A. Meissner and M. Olechowski, Phys. Rev. Lett. 86, 3708 (2001) hep-th/0009122; Y. M. Cho, I. Neupane, and P. S. Wesson, Nucl. Phys. B621, 388 (2002) hep-th/0104227.

[9] N. Deruelle and M. Sasaki, Prog. Theor. Phys. 110, 441 (2003) gr-qc/0306032.

[10] D. N. Spergel et al., Astrophys. J. Suppl. 148, 175 (2003) astro-ph/0302209; H. V. Peiris et al., Astrophys. J. Suppl. 148, 213 (2003) astro-ph/0302225.

[11] A. A. Starobinsky, Pis'ma Zh. Eksp. Teor. Fiz. 30, 719 (1979) (JETP Letters 30, 682); L. F. Abbott and M. B. Wise, Nucl. Phys. B244, 541 (1984).

[12] R. Crittenden, J. R. Bond, R. L. Davis, G. Efstathiou, and P. J. Steinhardt, Phys. Rev. Lett. 71, 324 (1993) astro-ph/9303014; M. Kamionkowski, A. Kosowsky, and A. Stebbins, Phys. Rev. Lett. 78, 2058 (1997) astro-ph/9611125; U. Seljak and M. Zaldarriaga, Phys. Rev. Lett. 78, 2054 (1997) astro-ph/9609169.

[13] D. Langlois, R. Maartens, and D. Wands, Phys. Lett. B489, 259 (2000) hep-th/0006007.

[14] S. C. Davis, Phys. Rev. D67, 024030 (2003) hep-th/0208205; E. Gravanis and S. Willison, Phys. Lett. B562, 118 (2003) hep-th/0209076; J. P. Gregory and A. Padilla, Class. Quantum Grav. 20, 4221 (2003) hep-th/0304250.

[15] C. Charmousis and J-F. Dufaux, Class. Quantum Grav. 19, 4671 (2002) hep-th/0202107.

[16] K. Maeda and T. Torii, Phys. Rev. D69, 024002 (2004) hep-th/0309152.

[17] J. E. Lidsey and N. Nunes, Phys. Rev. D67, 103510 (2003) astro-ph/0303168.

[18] This bound results from viewing the GB contribution to the action as a small correction, so that Eq. (2) holds, as one may expect from a string-inspired perspective. In this case, the GB term in the action would couple to the dilaton (in addition to compactification moduli), and the bound would be consistent with a small string coupling.

[19] S. Deser and A. Waldron, Phys. Lett. B508, 347 (2001) hep-th/0103255.

[20] A. Frolov and L. Kofman, hep-th/0209133

[21] T. Tanaka and X. Montes, Nucl. Phys. B582, 259 (2000) hep-th/0001092.

[22] U. Gen and M. Sasaki, Prog. Theor. Phys. 105, 591 (2001) gr-qc/0011078.

[23] J. Garriga and M. Sasaki, Phys. Rev. D62, 043523 (2000) hep-th/9912118.

[24] C. Charmousis, S. C. Davis, and J-F. Dufaux, J. High Energy Phys. 12, 029 (2003) hep-th/0309083.

[25] F. Cooper, A. Khare, and U. Sukhatme, Phys. Rep. 251, 267 (1995) hep-th/9405029.

[26] I. Cho, hep-th/0402125

[27] C. Charmousis and J-F. Dufaux, hep-th/0311267

[28] A. V. Frolov and L. Kofman, Phys. Rev. D69, 044021 (2004) hep-th/0309002.

[29] D. S. Gorbunov, V. A. Rubakov, and S. M. Sibiryakov, J. High Energy Phys. 10, 015 (2001) hep-th/0108017.

[30] T. Kobayashi, H. Kudoh, and T. Tanaka, Phys. Rev. D68, 044025 (2003) gr-qc/0305006.

[31] J. E. Lidsey, A. R. Liddle, E. W. Kolb, E. J. Copeland, T. Barreiro, and M. Abney, Rev. Mod. Phys. 69, 373 (1997).

[32] S. Tsujikawa and B. Gumjudpai, astro-ph/0402185

[33] G. Huey and J. E. Lidsey, Phys. Lett. B514, 217 (2001) astro-ph/0104006.

[34] G. Huey and J. E. Lidsey, Phys. Rev. D66, 043514 (2002) astro-ph/0205236.

[35] G. F. Giudice, E. W. Kolb, J. Lesgourgues, and A. Riotto, Phys. Rev. D66, 083512 (2002) hep-ph/0207145.

[36] D. Seery and A. N. Taylor, astro-ph/0309152 G. Calcagni, JCAP 11, 009 (2003) hep-ph/0310304.

[37] S. M. Leach and A. R. Liddle, Phys. Rev. D68, 123508 (2003) astro-ph/0306305; S. Tsujikawa and A. R. Liddle, JCAP 03, 001 (2004) astro-ph/0312162.

[38] R. Maartens, D. Wands, B. A. Bassett, and I. P. C. Heard, Phys. Rev. D62, 041301 (2000) hep-ph/9912464.

[39] D. Wands, K. A. Malik, D. H. Lyth, and A. R. Liddle, Phys. Rev. D62, 043527 (2000) astro-ph/0003278.

[40] S. Tsujikawa, M. Sami, and R. Maartens, astro-ph/0406078 